\title{
Lysostaphin Reduces the Production of Inflammatory Cytokines in Staphylococcus aureus Challenged Mice, and Prevents Systemic Shock
}

\author{
Clara Sei, Tanya Chanturiya*, James J. Mond and John F. Kokai-Kun*
}

Biosynexus Incorporated 9298 Gaither Rd. Gaithersburg, MD 20877, USA; ${ }^{*}$ National Institutes of Health NIDDK Building 3131 Center Drive Bethesda, MD 20892, USA

\begin{abstract}
Lysostaphin is being developed as a treatment for serious staphylococcal infections. Mice challenged with $S$. aureus produce inflammatory cytokines including, TNF- $\alpha$ and IL-6, and over-production of these cytokines can lead to shock and contribute to the lethality of staphylococcal infections. Two major components of the staphylococcal cell wall, peptidoglycan and lipoteichoic acid, are known to synergize to induce shock and organ failure in animal models, and we wished to determine whether the rapid lysostaphin-mediated degradation of peptidoglycan during treatment of systemic $S$. aureus infection could affect shock-associated parameters. We found that lysostaphin treatment of $S$. aureus-infected mice, which reduces bacteremia and organ infection, also reduced the serum levels of inflammatory cytokines and reversed the symptoms of $S$. aureus-induced shock. We compared the cytokine response of mice challenged with $S$. aureus to that of mice challenged with $S$. aureus and then treated with lysostaphin or nafcillin. Lysostaphin-treated mice, as compared with untreated mice or nafcillin-treated mice, had a blunted cytokine responses to $S$. aureus challenge. Core body temperature was used as a real time indicator for systemic shock in mice. Mice infected with $S$. aureus demonstrated a rapid drop in core body temperature, which was reversed by lysostaphin treatment. These studies demonstrated that lysostaphin treatment did not contribute to the induction of shock by rapidly releasing staphylococcal cell wall components, but rather it blunted the inflammatory cytokine response and ameliorated shock related symptoms.
\end{abstract}

Keyword: S. aureus, lysostaphin, shock, cytokine release.

\section{INTRODUCTION}

Bacterial infections are generally treated with antibiotics, but in certain cases antibiotic treatment is not indicated due to concerns that antibiotics could further exacerbate the clinical situation by lysing the bacteria and releasing toxic factors into the host. This is the case with certain food poisonings caused by E. coli and Vibrio parahaemolyticus where the concern is that antibiotic treatment could release bacterial toxins like Shiga toxins from the bacteria that may cause more harm to the host than the enteric infection alone $[1,2]$. This can also be the case with systemic gram negative infections during which, endotoxin, released by the bacteria, stimulates excessive cytokine production. This has been shown to be the case after treatment of infections caused by Pseudomonas aeruginosa or $E$ coli in the presence of amikacin, ceftazidime, imipenem, and ofloxacin $[3,4]$. In gram positive bacteria, free lipoteichoic acid (LTA) and peptidoglycan synergize to stimulate cytokine production through activation of TLR-2 and MyD88 and cause shocklike symptoms in animal models [5-9]. $\beta$-lactam antibiotics have been shown to stimulate the release of LTA from bacteria grown in vitro [10] and this may also occur in vivo during treatment of infection. Antibiotics that stimulate the release of bacterial components in infected hosts could lead to over-expression of cytokines and result in shock

*Address correspondence to this author at the Biosynexus Incorporated 9298 Gaither Rd. Gaithersburg, MD 20877, USA; Tel: (301) 987-1172; Fax: (301) 944-2141; E-mail: johnkun@biosynexus.com particularly if these bacterial components are released rapidly and in large quantities.

Lysostaphin is a highly active, lytic, anti-staphylococcal enzyme which is being developed as a treatment for serious staphylococcal infections, and has demonstrated efficacy in animal models of staphylococcal infection [11-15]. It is a glycyl-glycine endopeptidase which rapidly lyses staphylococci by cleaving the pentaglycine cross-bridge in the staphylococcal cell wall [16]. A single dose of lysostaphin will virtually eliminate the blood-borne Staphylococcus aureus from a bacteremic animal by lysing the bacteria [12]. Because lysostaphin rapidly lyses staphylococci which releases bacterial components like LTA and peptidoglycan into the system, the concern is that treatment of a systemic staphylococcal infection with lysostaphin could lead to cytokine over-expression and systemic shock.

In this study, we demonstrated that treatment of S. aureuschallenged mice with lysostaphin reduced the expression of inflammatory cytokines, and blunted shock-like symptoms as measured by a drop in core body temperature.

\section{MATERIALS AND METHODOLOGY}

\section{Materials}

Recombinant mature lysostaphin was produced by fermentation in E. coli and purified to homogeneity by Avecia (Stanstead, UK) under contract to Biosynexus Incorporated (Gaithersburg, MD) [17]. Purified lysostaphin 
was formulated in $\mathrm{pH}$ 6.5, phosphate buffered saline (PBS) for storage at $-70^{\circ} \mathrm{C}$ until used. Nafcillin was purchased from Sigma (St. Louis, MO). All reagents were endotoxin-free.

\section{Preparation of $\boldsymbol{S}$. aureus for Challenge}

S. aureus was prepared for intravenous challenge of mice as previously described [12].

\section{Analysis of Cytokine Response to $S$. aureus in Treated Mice}

All animal experiments were carried out in accordance with Biosynexus' Institutional Animal Care and Use Committee approval. Groups of 5, six week-old, female Balb/c mice (Harlan, Indianapolis, IN) were challenged with $2 \times 10^{7}$ of a well characterized $S$. aureus strain, ATCC 49521 (ATCC, Manassas, VA) by injection in the tail vein [12]. Control animals were not challenged with $S$. aureus. Either immediately after challenge or 24 hours after challenge, the mice were treated with intravenous injections of either lysostaphin $(20 \mathrm{mg} / \mathrm{kg})$ or nafcillin $(50 \mathrm{mg} / \mathrm{kg})$ or a combination of both in $200 \mu$ l of PBS, pH 7.2. Blood samples were collected at 2, 6 and 24 hours post-treatment. Red blood cells were separated from the serum by centrifugation, and the serum was stored at $-70^{\circ} \mathrm{C}$ until analyzed. Serum samples were analyzed for TNF- $\alpha$ and IL-6 as indicators of innate and acute phase responses. TNF- $\alpha$ and IL-6 levels were analyzed by ELISA using the eBioscience (San Diego, CA) Mouse TNF- $\alpha$ and Mouse IL-6 ELISA Ready-SET-Go! Briefly, each sample of mouse serum was diluted between 3 and 81-fold for TNF- $\alpha$ and 25 and 400fold for IL- 6 and the capture ELISA assay was performed on triplicate samples as per the manufacturer's instructions. The concentration of each cytokine at each time point was determined by comparison with ELISA responses to a reference standard curve of control cytokines provided with the kit. Data presented are representative of two independent experiments.

\section{Measuring Hemodynamic Shock in Mice Challenged with $S$. aureus}

The core body temperature of mice was used as a real time measure of hemodynamic shock due to $S$. aureus challenge. In this model, six week-old female CF-1 mice (Harlan, Indianapolis, IN) were implanted with an IPTT-300 microchip transponders (Bio Medic Data Systems, Seaford DE) 24 hours prior to bacterial challenge. On the day of challenge, an overnight culture of $S$. aureus was prepared for intravenous challenge. A baseline core body temperature was established for each mouse using the DAS-5002 Notebook Scanner system (Bio Medic Data Systems), and then four groups of five mice each were intravenously challenged with either, $3.6 \times 10^{8} \mathrm{~S}$. aureus (three groups) or $3.6 \times 10^{8} \mathrm{~S}$. aureus that had been pretreated for 30 minutes with $100 \mu \mathrm{g}$ of lysostaphin (equivalent to $5 \mathrm{mg} / \mathrm{kg}$ lysostaphin dose). One of the three groups of mice challenged with whole $S$. aureus then received an intraperitoneal dose of lysostaphin of $5 \mathrm{mg} / \mathrm{kg}$ immediately after bacterial challenge. This group as well as another group of mice challenged with whole $S$. aureus then received i.p. lysostaphin injections $(5 \mathrm{mg} / \mathrm{kg})$ at two hours after bacterial challenge and then b.i.d. for three additional days. Starting at 30 minutes after $S$. aureus challenge and then every 10 minutes for 4 hours, the core body temperatures of the mice were monitored on the day of challenge and surviving mice were also monitored three times a day on subsequent days. Blood was also drawn from all surviving challenged mice on a daily basis to monitor bacteremia [12]. Data presented are representative of three independent experiments.

\section{Statistical Analysis}

Data sets were analyzed for statistically significant differences by analysis of variance with a post-hoc test for comparing multiple tests. Differences were considered significant if $p \leq 0.05$ for both the overall ANOVA and the pair-wise comparisons.

\section{RESULTS}

Mice Challenged with $S$. aureus and then Treated with Lysostaphin Expressed less Inflammatory Cytokines then Mice Challenged with $S$. aureus Alone

When mice were challenged with $2 \times 10^{7} \mathrm{~S}$. aureus, TNF$\alpha$ and IL- 6 could be detected in the serum within two hours (Fig. 1). TNF- $\alpha$ concentrations peaked at six hours post challenge and then dropped to slightly above detection at 24 hours post- challenge, while IL-6 concentrations remained high from 2 hours post-challenge through at least 24 hours post-challenge. Serum from unchallenged mice had no detectable TNF- $\alpha$ or IL- 6 at any time point (data not shown). When mice challenged with $S$. aureus were treated with nafcillin immediately post-challenge, they displayed a significant increase in TNF- $\alpha$ concentration in their serum at 2 hours and were not different than control serum at the later time points. In marked contrast, when lysostaphin was administered immediately after $S$. aureus challenge TNF- $\alpha$ serum concentrations were significantly reduced as compared with both control mice as well as nafcillin-treated mice at all time points (Fig. 1). Further, lysostaphin treatment significantly reduced IL-6 expression versus control and nafcillin-treated mice at 6 and 24 hours postchallenge. Unchallenged mice treated with nafcillin or lysostaphin alone did not have detectable levels of cytokines in their serum at any time point (data not shown).

When $S$ aureus-challenged mice were treated with nafcillin or lysostaphin 24 hours post-challenge, a different cytokine response was seen than described above. As noted previously, at 24 hours post challenge, TNF- $\alpha$ concentrations were already low and were not affected significantly by nafcillin or lysostaphin treatment (Fig. 2). Only the combination treatment of lysostaphin and nafcillin at 24 hours post-challenge led to a significant reduction in TNF- $\alpha$ post-treatment. IL- 6 concentrations remained elevated beyond 24 hours post-challenge. Lysostaphin or nafcillin treatment at 24 hours post-challenge did not significantly change the concentration of IL- 6 within 3 hours after treatment, but both treatments significantly reduced IL- 6 concentrations by 6 hours post-treatment (Fig. 2). Further, lysostaphin or lysostaphin combined with nafcillin significantly reduced the concentration of IL- 6 at 6 hours post-treatment as compared with nafcillin alone. 


\section{TNF $\alpha$}
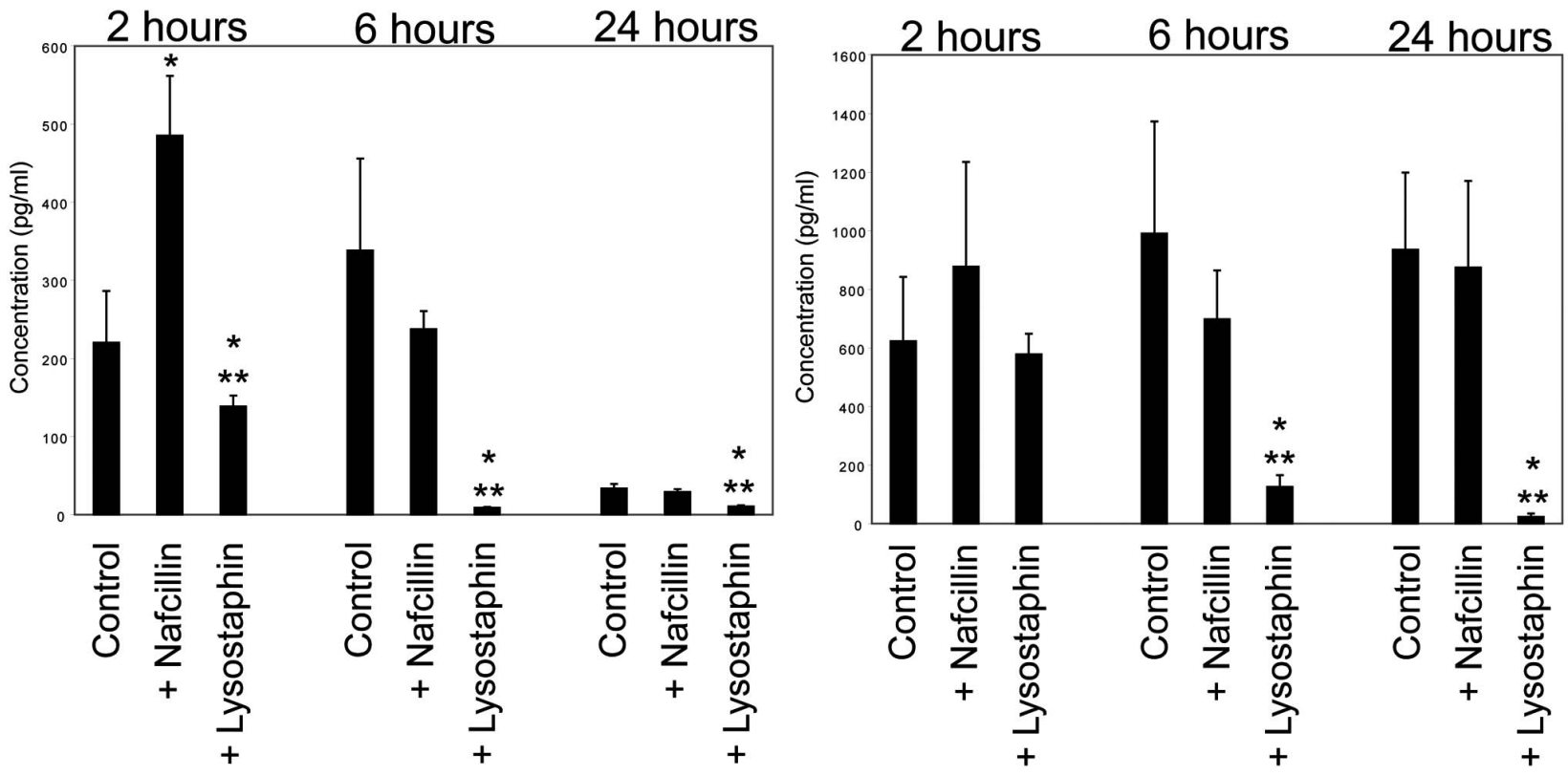

Fig. (1). Lysostaphin reduced the expression of inflammatory cytokines in $S$. aureus challenged mice when administered immediately after challenge. Groups of 5 mice each were challenged $2 \times 10^{7} \mathrm{~S}$. aureus and then immediately after challenge received an i.v. injection to of either PBS (Control), $50 \mathrm{mg} / \mathrm{kg}$ of nafcillin (+Nafcillin) or $20 \mathrm{mg} / \mathrm{kg}$ of lysostaphin (+Lysostaphin). Blood samples were then taken from the mice at 2, 6 and 24 hours and analyzed for TNF- $\alpha$ and IL-6. The graphs display the mean serum concentration in pg/ml for the two cytokines at the various time points as indicated, \pm standard deviation. The single asterisks $(*)$ indicate that the cytokine concentrations were significantly different than the Control group, while the double asterisks $(* *)$ indicate that the cytokine concentrations were significantly different than the +Nafcillin group.

TNF $\alpha$

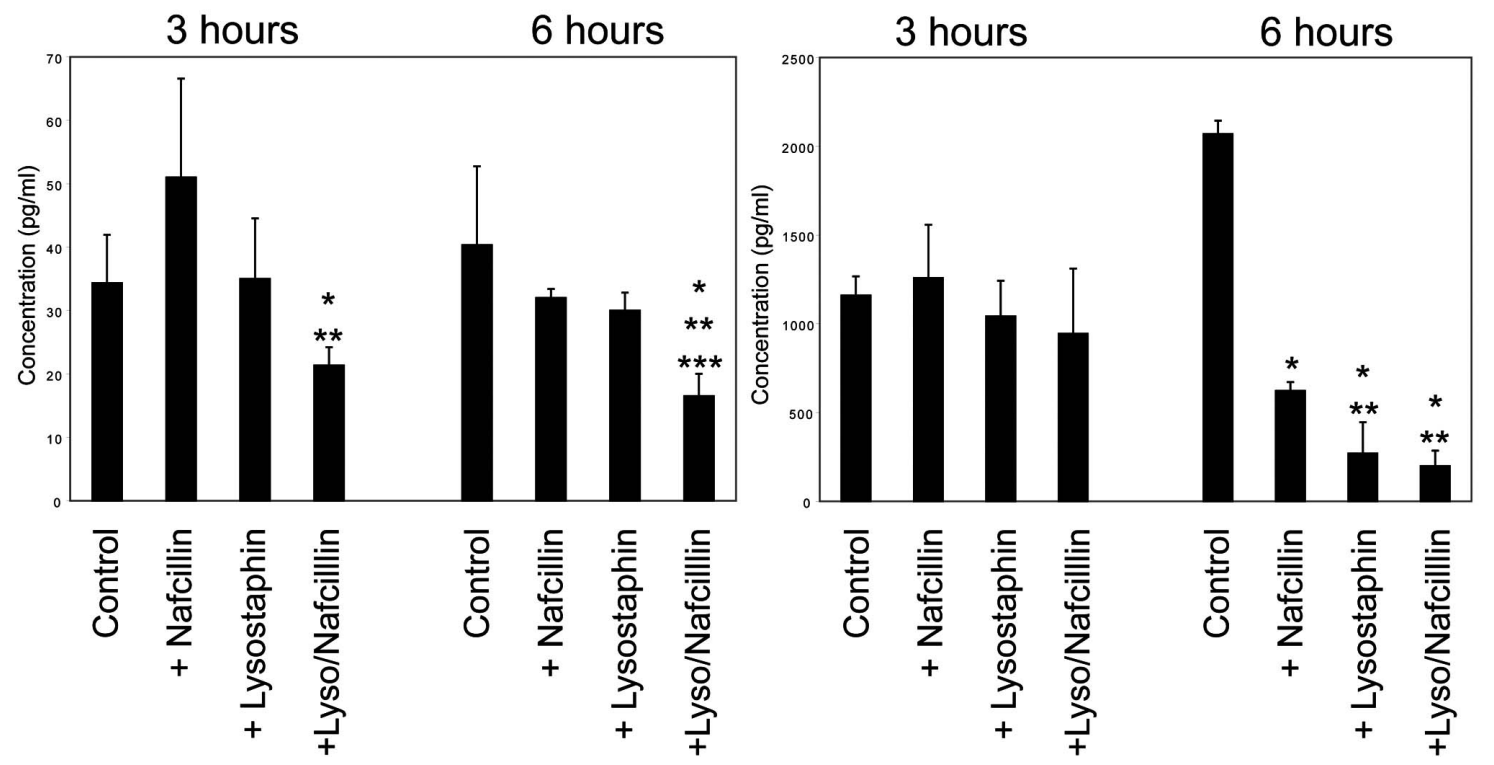

Fig. (2). Lysostaphin was less effective in reducing inflammatory cytokines when delivered 24 hours after the challenge. Groups of 5 mice were challenged $2 \times 10^{7} \mathrm{~S}$. aureus and then 24 hours after challenge received an i.v. injection to of either PBS (Control), 50mg/kg of nafcillin (+Nafcillin), 20mg/kg of lysostaphin (+Lysostaphin) or lysostaphin $(20 \mathrm{mg} / \mathrm{kg})$ and nafcillin $(50 \mathrm{mg} / \mathrm{kg}) \mathrm{combined}(+\mathrm{Lyso} / \mathrm{Nafcillin})$. Blood samples were then taken from the mice at 3 and 6 hours and analyzed for TNF- $\alpha$ and IL-6. The graphs display the mean serum concentration in $\mathrm{pg} / \mathrm{ml}$ for the two cytokines at the various time points as indicated, \pm standard deviation. The single asterisks $(*)$ indicate that the cytokine concentrations were significantly different than the control group, while the double asterisks (**) indicate that the concentrations were significantly different than the + Nafcillin group. Three asterisks $(* * *)$ indicate that the concentration was significantly different than the +Lysostaphin group. 
Lysostaphin Blunted the Initial Temperature Drop Caused by $S$. aureus Challenge and Prevented the Animals from Progressing to Hemodynamic Shock Due to Sepsis

When mice were challenged with $3.6 \times 10^{8} \mathrm{~S}$. aureus, there was an almost immediate drop in core body temperature of $\sim 5^{\circ} \mathrm{C}$ which then recovered in all mice about one hour after challenge (Fig. 3, insert). Immediate administration of $5 \mathrm{mg} / \mathrm{kg}$ lysostaphin after bacterial challenge blunted this temperature drop, resulting in a core body temperature drop of only $\sim 2^{\circ} \mathrm{C}$. To determine if staphylococci pre-lysed with lysostaphin would have this same effect, mice were challenged with $S$. aureus that had been treated in vitro with lysostaphin. No core body temperature drop was seen in these mice (Fig. 3, insert).

Consistent with previous results [12], all control animals challenged with $S$. aureus developed bacteremia (average $\sim 12,000 \mathrm{CFU} / \mathrm{ml}$ of blood) at 24 hours post challenge, while lysostaphin-treated animals averaged $<100 \mathrm{CFU} / \mathrm{ml}$ of blood. Lysostaphin-treated animals were free of bacteremia by 48 hours post challenge.

Within 24 hours of $S$. aureus challenge, untreated, control mice began to lose their ability to maintain their core body temperatures, with temperatures dropping an average of $10^{\circ} \mathrm{C}$ prior to sacrificing the animals at 28 hours post challenge (Fig. 3). Lysostaphin treatment prevented this drop in core body temperature. In a separate experiment, $S$. aureus-challenged mice that had reduced core body temperature at 24 hours post challenge were treated with $20 \mathrm{mg} / \mathrm{kg}$ of lysostaphin, but treatment at this late time point was unable to reverse the hemodynamic shock and multiorgan failure that had occurred due to the high challenge dose of $S$. aureus and the mice had to be sacrificed (data not shown).

\section{DISCUSSION}

During infection, the host recognizes the invading pathogen as foreign through a number of mechanisms. The Toll-like receptors (TLRs) play an important role in this recognition as do other pathways of the innate immune system which recognize foreign molecular patterns (PAMPS) and activate the innate immune system to release proinflammatory cytokines. This process can be dysregulated and culminate in sepsis [18] which constitutes the systemic over-response to infection and is a major cause of morbidity and mortality in patients admitted to the intensive care unit with mortality often reaching 50\% [19]. The septic response

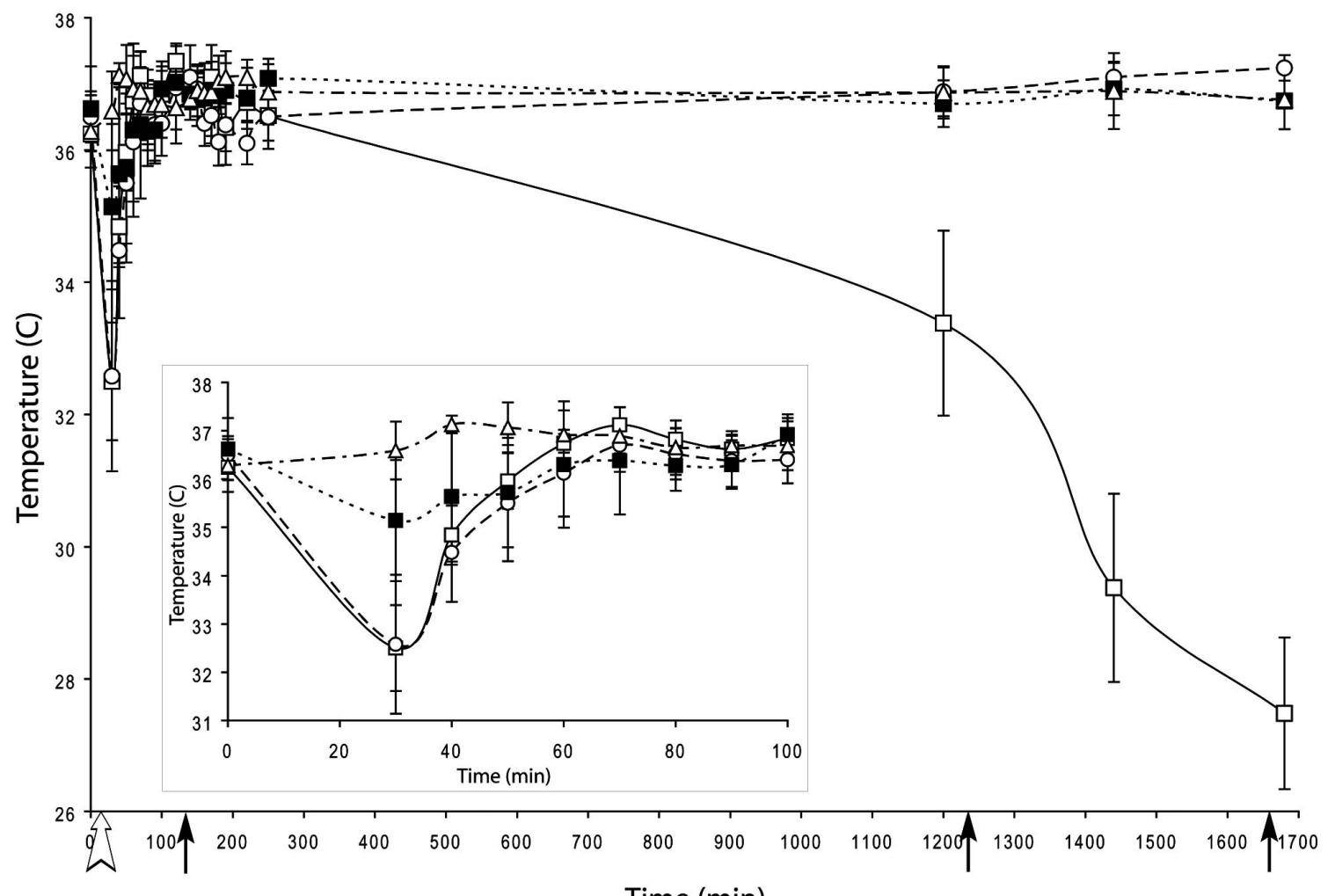

Time (min)

Fig. (3). Lysostaphin blunted the initial temperature drop and prevented the more delayed loss of hemodynamic stability in $S$. aureus-challenged mice. Groups of five mice were challenged with either $S$. aureus (closed squares, open squares or open circles) or $S$. aureus that had been pretreated with lysostaphin (open triangles). The mice challenged with whole $S$. aureus were then treated with either lysostaphin (open circles and closed squares) or nothing (control untreated mice (open squares)). Lysostaphin treatments (indicated by the closed arrows) were once on the day of challenge and twice on the following day. One group of mice (closed squares) also received an additional lysostaphin treatment immediately after $S$. aureus challenge (open arrow). Core body temperature was monitored at various time points as shown on the figure. The inset graph displays the early time points through 100 minutes post-challenge. The graph is the mean of the core body temperature of five animals \pm the standard deviation versus time in minutes post $S$. aureus challenge. The data are representative of three independent experiments. 
is marked by the sequential generation of pro-inflammatory cytokines among the most important of which is TNF- $\alpha$ [20]. The pro-inflammatory effects of these cytokines are inhibited by various receptor antagonists and anti-inflammatory cytokines including IL-10 and transforming growth factor- $\beta$ [20]. Modulation of the activity of both proand anti-inflammatory cytokines as well as modulation of regulatory cells which control these processes in order to improve outcome in patients with sepsis has been the subject of multiple clinical interventions and has determined that reducing cytokine over-production during infections would be desirable [20]. In a recent paper, Bannerjee et al. demonstrated that mice that had reduced levels of a number of different inflammatory cytokines had improved survival for staphylococcal pneumonia as compared with wild type mice [21].

When $S$. aureus infects a host, it produces factors that interact with the immune system, some of these factors induce a protective response by the host and some of these factors are beneficial to the bacteria through modulation of the immune response [22]. Two of the most prevalent components in staphylococci that play a role in this interaction are peptidoglycan and LTA [23]. These two components comprise much of the cell wall of the bacteria [24]. Free LTA binds to TLR2, and in conjunction with interaction with $\mathrm{C} 5 \mathrm{aR}$ [8], induces inflammatory cytokine production which includes TNF- $\alpha$ and IL-6 [25] with a pattern of immunostimulation and proinflammatory mediator production that is similar in many respects to the response induced by LPS from gram negative bacteria [26]. Peptidoglycan is also recognized by TLR2 [7], and in combination with LTA, acts synergistically to cause shock and multiple organ failure [5] through the production of nitric oxide [6].

S. aureus-challenged mice develop bacteremia and have high levels of $S$. aureus in their spleen, heart and liver by 24 hours post challenge [12]. A single dose of $5 \mathrm{mg} / \mathrm{kg}$ lysostaphin clears bacteremia by rapidly lysing the infecting bacteria, and with three days of dosing, also clears infected organs [12]. The rapid lytic action of lysostaphin on staphylococci depends on degradation of the peptidoglycan [24]. Lysostaphin binds directly to the cross-linked peptidoglycan in the cell wall envelop of $S$. aureus [27], and enzymatically cleaves the pentaglycine oligopeptides [28] between the third and fourth glycine [29]. This enzymatic cleavage rapidly degrades the peptidoglycan leading to the formation of osmotically fragile cells which swell and quickly lyse [30] releasing peptidoglycan fragments and LTA into the surrounding media[27]. It has been found that synthetic peptidoglycan partial structures do not stimulate TLR2 in vitro suggesting that there may be some minimal three dimensional structural requirement for cytokine stimulation [31]. This may partially explain why treatment of $S$. aureus-challenged mice with lysostaphin which releases LTA and peptidoglycan actually blunts cytokine expression rather than enhancing it (Fig. 1). The peptidoglycan fragments which are released by lysostaphin digestion may be lacking a critical structure to induce cytokine formation.

Another possible explanation for why treatment of $S$. aureus-challenged animals with lysostaphin blunts cytokine expression may be found in a recent publication by Ip et al.
[9]. In this publication, it was demonstrated that prior to TLR-dependent cytokine production, whole staphylococci must be engulfed and delivered into acidic phagosomes where acid-activated host enzymes digest the internalized bacteria to liberate otherwise cryptic bacterial-derived ligands that initiate the cytokine response from vacuoles. In our study, lysostaphin was delivered systemically to the mice rapidly lysing non-phagocytosed staphylococci, but since lysostaphin does not cross cell membranes [32], it lyses only extra-cellular $S$. aureus. The bacterial factors that are released during this lysis are thus diluted throughout the system and likely do not reach concentrations comparable to those found within phagosomes to allow signaling as described by Ip et al. This is supported by the finding that if lysostaphin treatment is delayed until 24 hours post challenge, the blunting of the cytokine response is diminished in comparison to the reduced response seen when lysostaphin is given shortly after challenge (Fig. 2). Many of the $S$. aureus would have already been phagocytosed by 24 hours post challenge and are thus signaling through TLR2 following degradation in the phagosomes.

In this study, we also used core body temperature of the mice as a real-time indicator of septic shock [33]. Within 30 minutes after infusion of $S$. aureus, mice core temperature dropped by $\sim 5^{\circ} \mathrm{C}$ and then fully recovered by $60-70$ minutes post-challenge (Fig. 3). In vitro pretreatment of the $S$. aureus with lysostaphin prevented this core temperature drop, while immediate treatment of mice with lysostaphin following bacterial challenge blunted the initial temperature drop and prevented the delayed temperature drop as well. This is consistent with the rapid recognition of intact bacteria by the mouse immune system leading to phagocytosis, phagolysosome formation and induction of cytokines inducing temperature drop. Lysostaphin pre-digestion of the bacteria rendered them inactive in terms of inducing systemic shock in this model.

As the $S$. aureus infection progressed in the challenged animals, they lost their capacity to regulate their core body temperature (Fig. 3), and over the next $\sim 27$ hours post infection the core temperatures dropped to $<28^{\circ} \mathrm{C}$ prior to the animals being sacrificed. Lysostaphin treatment of $S$. aureuschallenged mice at 2 hours post challenge reversed this temperature drop Consistent with previously reported data [12], two additional lysostaphin treatments also cleared the infection in all animals (data not shown). In separate experiments, $S$. aureus-challenged mice that had begun to show rapid decline in body temperature by 20 hours postchallenge were treated with a bolus of lysostaphin, but, this treatment in moribund animals failed to save the mice most likely because they had already progressed to multi organ failure (data not shown).

\section{CONCLUSION}

When considering how rapidly lysostaphin lyses $S$. aureus and clears infection, a concern could be raised that the rapid release of cell contents into the infected host could lead to massive cytokine release and septic shock. In the present study we have demonstrated that lysostaphin treatment of $S$. aureus-challenged animals blunted both the immediate $S$. aureus-induced release of cytokines as well as the more delayed septic shock associated with $S$. aureus 
infection. This finding supports the use of lysostaphin for treatment of serious $S$. aureus infections in humans who are susceptible to interventions that would result in inflammatory over-responses during staphylococcal infections.

\section{CONFLICT OF INTEREST}

Funding for these studies was provided by Biosynexus Incorporated. All authors are, or formerly were, employees and stock holders of Biosynexus Incorporated, and Biosynexus is in the process of developing lysostaphin for commercial use.

\section{ACKNOWLEDGEMENTS}

We would like to thank Julio Canas and Elizabeth Mendez for excellent technical assistance.

\section{REFERENCES}

[1] Wong CS, Jelacic S, Habeeb RL, Watkins SL, Tarr PI. The risk of the hemolytic-ueremic syndrome after antibiotic treatment of Escherichia coli O157:H7 infections. N Engl J Med 2000; 342:1930-6.

[2] Gilbert DN, Moellering RC, Sande MA. The Sandford Guide to Antimicrobial Therapy. 33rd ed. Hyde Park, VT: Antimicrobial Therapy Inc. 2003.

[3] Morrison DC, Bucklin SE. Evidence for antibiotic-mediated endotoxin release as a contributing factor to lethality in experimental gram-negative sepsis. Scand J Infect Dis Suppl 1996; 101: 3-8.

[4] Holzheimer RG. Antibiotic induced endotoxin release and clinical sepsis: a review. J Chemother 2001; Spec No 1: 159-72.

[5] DeKimpe SJ, Kengatharan M, Thierermann C, Vane JR. The cell wall components peptidoglycan and lipoteichoic acid from Staphylococcus aureus act in synergy to cause shock and multiple organ failure. Proc Nat Acad Sci USA 1995; 92: 10359-63.

[6] Kengatharan KM, DeKimpe S, Robson C, Foster SJ, Thiermann C. Mechanism of gram-positive shock: identification of peptidoglycan and lipoteichoic acid moieties essential in the induction of nitric oxide synthase, shock, and multiple organ failure. J Exp Med 1998; 188: 305-15.

[7] Janssens S, Beyaert R. Role of toll-like receptors in pathogen recognition. Clin Microbiol Rev 2003; 16: 637-46.

[8] Mullaly SC, Kubes P. The role of TLR2 in vivo following challenge with Staphylococcous aureus and prototypic ligands. J Immunol 2006; 177: 8154.

[9] Ip WKE, Sokolovska A, Charriere GM, Boyer L, Dejardin S, Cappillino MP, et al. Phagocytosis and phagosome acidification are required for pathogen processing and MyD88-dependent responses to Staphylococcus aureus. J Immunol 2010; 184: 7071-81.

[10] Pollack JH, Ntamere AS, Neuhaus FC. D-alanyl-lipoteichoic acid Lactobacillus casei: secretion of vesicles in response to benzylpenicillin. J Gen Micro 1992; 138: 849-59.

[11] Kokai-Kun JF, Chanturiya T, Mond JJ. Lysostaphin eradicates established Staphylococcus aureus biofilms in jugular vein catheterized mice. J Antimicrob Chemother 2009; 64: 94-100.

[12] Kokai-Kun JF, Chanturiya T, Mond JJ. Lysostaphin as a treatment for systemic Staphylococcus aureus infection in a mouse model. J Antimicrob Chemother 2007; 60: 1051-9.

[13] Kokai-Kun JF, Walsh SM, Chanturiya T, Mond JJ. Lysostaphin cream eradicates Staphylococcus aureus nasal colonization in a cotton rat model. Antimicrob Agents Chemother 2003; 47: 1589-97.
[14] Climo MW, Patron RL, Goldstein BP, Archer GL. Lysostaphin treatment of experimental methicillin-resistant Staphylococcus aureus aortic valve endocarditis. Antimicrob Agents Chemother 1998; 42: 1355-60.

[15] Goldberg LM, DeFranco JM, Watanakunakorn C, Hamburger M. Studies in experimental staphylococcal endocarditis in dogs. VI. Treatment with lysostaphin. Antimicrob Agents Chemother 1967; 7: 45-53.

[16] Schindler CA, Schuhardt VT. Lysostaphin: a new bacteriolytic agent for the staphylococci. Proc Nat Acad Sci USA 1964; 51: 414-21.

[17] McCoy M. Killing staph together: Start-up Biosynexus places fermentation project in newly started Avecia facility. Chem Eng News 2004; 82: 36-40.

[18] Weighardt H, Holzmann B. Role of Toll-like receptor responses for sepsis pathogenesis. Immunobiol 2007; 212: 715-22.

[19] Winters BD, Eberlein M, Leung J, Needham DM, Pronovost PJ, Sevransky JE. Long-term mortality and quality of life in sepsis: a systematic review. Crit Care Med 2010; 38: 1276-38.

[20] Zanotti S, Kumar A, Kumar A. Cytokine modulation in sepsis and septic shock. Expert Opin Investig Drugs 2002; 11: 1061-75.

[21] Banerjee A, Stevenaert F, Pande K, et al. Modulation of paired immunoglobulin-like type 2 receptor signaling alters the host response to Staphylococcus aureus-induced pneumonia. Immunity 2010; 78: 1353-63.

[22] DeLeo FR, Diep BA, Otto M. Host defense and pathogenesis in Staphylococcus aureus infections. Infect Dis Clin North Am 2009; 23: $17-34$

[23] Neuhaus FC, Baddiley J. A continuum of anionic charge: structures and functions of D-alanyl-teichoic acids in gram-positive bacteria. Microb Molec Biol Rev 2003; 67: 686-723.

[24] Tomasz A. The staphylococcal cell wall. In: Fischetti VA, Novick RP, Ferretti JJ, Portnoy DA, Rood JI, editors. Gram Positive Pathogens. Washington DC: ASM Press 2000.

[25] Ellingsen EA, Morath S, Flo TH, et al. Induction of cytokine production in human $\mathrm{T}$ cells and monocytes by highly purified lipoteichoic acid: involvement of Toll-like receptors and CD-14. Med Sci Monit 2002; 8: BR149-BR56.

[26] Kimbrell MR, Warshakoon H, Cromer JR, et al. Comparison of the immunostimulatory and proinflammatory activities of candidate Gram-positive endotoxins, lipoteichoic acid, peptidoglycan, and lipopeptides, in murine and human cells. Immunol Lett 2008; 118: $132-41$.

[27] Grundling A, Schneewind O. Cross-linked peptidoglycan mediates lysostaphin binding to the cell wall envelop of Staphylococcus aureus. J Bacteriol 2006; 188: 2463-72.

[28] Browder HP, Zygmunt WA, Young JR, Travormina PA. Lysostaphin: enzymatic mode of action. Biochem BioPhys Res Comm 1965; 19: 383-9.

[29] Schneewind OA, Fowler A, Faull KF. Structure of the cell wall anchor of surface proteins in Staphylococcus aureus. Science 1995; 268: 103-6.

[30] Francius G, Domenech O, Mingeot-Leclercq MP, Dufrene YF. Direct observation of Staphylococcus aureus cell wall digestion by lysostaphin. J Bacteriol 2008; 190: 7904-9.

[31] Fujimoto Y, Inamura S, Kawasaki A, et al. Chemical synthesis of peptidoglycan fragments for elucidation of the immunostimulating mechanism. J Endotoxin Res 2007; 13: 189-96.

[32] Craven N, Anderson JC. The selection in vitro of antibiotics with activity against intracellular $S$. aureus. J Vet Pharmacol Therap 1980; 3: 221-6.

[33] Oliveira-Pelegrin GR, Branco LG, Rocha MJ. Central NO-cGMP pathway in thermoregulation and survival rate during polymicrobial sepsis. Can J Physiol Pharmacol 2010; 88: 113-20.

This is an open access article licensed under the terms of the Creative Commons Attribution Non-Commercial License (http://creativecommons.org/licenses/ by-nc/3.0/) which permits unrestricted, non-commercial use, distribution and reproduction in any medium, provided the work is properly cited. 